\title{
Plaque characterization in ex vivo MRI evaluated by dense 3D correspondence with histology
}

\author{
Arna van Engelen $^{* a}$, Marleen de Bruijne ${ }^{\mathrm{a}, \mathrm{b}}$, Stefan Klein ${ }^{\mathrm{a}}$, Hence Verhagen ${ }^{\mathrm{c}}$, Harald Groen ${ }^{\mathrm{d}, \mathrm{e}, \mathrm{f}}$, \\ Jolanda Wentzel ${ }^{\mathrm{d}}$, Aad van der Lugt ${ }^{\mathrm{e}}$, and Wiro Niessen ${ }^{\mathrm{a}, \mathrm{g}}$ \\ ${ }^{a}$ Biomedical Imaging Group Rotterdam, Departments of Radiology \& Medical Informatics, Erasmus \\ MC, Rotterdam, The Netherlands \\ ${ }^{b}$ Image Group, Department of Computer Science, University of Copenhagen, Denmark \\ 'Department of Vascular Surgery, Erasmus MC, Rotterdam, The Netherlands \\ ${ }^{\mathrm{d}}$ Department of Biomedical Engineering, Erasmus MC, Rotterdam, The Netherlands \\ ${ }^{e}$ Department of Radiology, Erasmus MC, Rotterdam, The Netherlands \\ ${ }^{\mathrm{f}}$ Department of Nuclear Medicine, Erasmus MC, Rotterdam, The Netherlands \\ ${ }^{g}$ Imaging Science and Technology, Faculty of Applied Sciences, Delft University of Technology, \\ Delft, The Netherlands
}

\begin{abstract}
Automatic quantification of carotid artery plaque composition is important in the development of methods that distinguish vulnerable from stable plaques. MRI has shown to be capable of imaging different components noninvasively. We present a new plaque classification method which uses 3D registration of histology data with ex vivo MRI data, using non-rigid registration, both for training and evaluation. This is more objective than previously presented methods, as it eliminates selection bias that is introduced when 2D MRI slices are manually matched to histological slices before evaluation.

Histological slices of human atherosclerotic plaques were manually segmented into necrotic core, fibrous tissue and calcification. Classification of these three components was voxelwise evaluated. As features the intensity, gradient magnitude and Laplacian in four MRI sequences after different degrees of Gaussian smoothing, and the distances to the lumen and the outer vessel wall, were used. Performance of linear and quadratic discriminant classifiers for different combinations of features was evaluated. Best accuracy $(72.5 \pm 7.7 \%)$ was reached with the linear classifier when all features were used. Although this was only a minor improvement to the accuracy of a classifier that only included the intensities and distance features $(71.6 \pm 7.9 \%)$, the difference was statistically significant (paired t-test, $p<0.05)$. Good sensitivity and specificity for calcification was reached ( $83 \%$ and $95 \%$ respectively), however, differentiation between fibrous (sensitivity $85 \%$, specificity $60 \%$ ) and necrotic tissue (sensitivity $49 \%$, specificity $89 \%$ ) was more difficult.
\end{abstract}

Keywords: Atherosclerosis, segmentation, quantification, pattern recognition

\section{INTRODUCTION}

Cerebral infarction is one of the most important causes of death and the greatest cause of disability in the Western world. Approximately $20 \%$ to $30 \%$ of the infarcts can be related to carotid artery atherosclerosis ${ }^{1}$. The development of an atherosclerotic plaque in the vessel wall leads to stenosis of the vessel lumen. It is current opinion that atherosclerotic plaque rupture plays an important role in acute events, like transient ischemic accidents (TIAs) and stroke. As not all plaques rupture, it is clinically important to determine the risk-of-rupture of individual plaques. Evidence is present that plaque vulnerability depends mainly on plaque composition rather than on plaque size or degree of stenosis ${ }^{2,3}$. A large lipid or necrotic core ${ }^{2}$ covered by a thin fibrous cap ${ }^{4}$ is associated with a high risk of rupture, while fibrous tissue and calcifications $^{3}$ have a stabilizing effect. Magnetic resonance imaging (MRI) allows visualization of the different plaque components. Both in vivo ${ }^{5-7}$ and ex vivo ${ }^{8,9}$, the accuracy of MRI in the detection of different plaque components has been demonstrated compared to histological sections. The relative intensity patterns for plaque components in different MRI

\footnotetext{
*E-mail: a.vanengelen@erasmusmc.nl
}

Medical Imaging 2011: Computer-Aided Diagnosis, edited by Ronald M. Summers, Bram van Ginneken, Proc. of SPIE Vol. 7963, 796329 - (C) 2011 SPIE · CCC code: 0277-786X/11/\$18 · doi: 10.1117/12.878007 
contrasts showed similar behavior in vivo and ex vivo ${ }^{10}$. Automatic segmentation and quantification of these plaque components in MR images is therefore relevant for risk stratification.

Automated plaque segmentation methods have been presented before by several authors ${ }^{10-17}$. In MRI images from ex vivo carotid specimens, segmentations of different components have been obtained with supervised classification using a maximum likelihood classifier ${ }^{12}$ or a minimum distance to means classifier ${ }^{10}$, and with unsupervised k-means clustering $^{15}$. The probability of misclassification with the assumption of either a multidimensional normal distribution or nonparametric Parzen window estimation, was determined by investigating the underlying probabilistic relationships between the classes ${ }^{17}$. On coronary specimens k-means clustering, in combination with an iterative approach to take into account the classification of neighboring voxels, has been presented ${ }^{14}$. In the analysis of in vivo MRI imaging data, similar approaches have been introduced. A comparison of performance of a standard Bayesian classifier, k-nearest neighbor, a feedforward neural network, and a Bayesian classifier in combination with a Parzen classifier to consider information from neighboring pixels, was performed ${ }^{13}$. Based on the results of this study, the standard Bayesian classifier was preferred for the quantification of calcifications, fibrous and lipid tissue and intraplaque hemorrhage ${ }^{13}$. In addition, fuzzy clustering followed by contour smoothing ${ }^{11}$, and a maximum likelihood Bayesian method that besides intensities also included the distance of each voxel to the lumen and outer vessel wall ${ }^{16}$ have been investigated. Except for this last method and the methods that take into account information from neighboring voxels ${ }^{11,13}$, all other methods mentioned above are based on MRI intensities of the voxel to be classified only.

In general, these studies reported reasonable to good results for the classification of different plaque components. In the evaluation of classifiers, results are often evaluated by comparing the resulting areas to manual delineations in the MRI for in vivo imaging ${ }^{11}$, or by comparing the measured volumes with the volumes obtained from histology ${ }^{13-15}$. Some

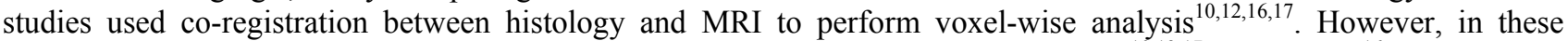
studies corresponding slices were manually selected prior to registration, both ex vivo ${ }^{10,12,17}$ and in vivo ${ }^{16}$, where in the latter the contours were drawn in the MRI with histology as reference. This manual selection may have induced a positive bias of classification accuracy as slices showing clear correspondence between histology and MRI can be selected and matched. In addition, it is not possible to account for differences in slice orientation between the histological and MRI slices.

This study presents a new method for segmentation of calcifications, fibrous and necrotic tissue in MRI images obtained from ex vivo carotid specimens. A framework for 3D registration was used for classifier training and evaluation to allow for more objective comparison between histology and MRI. Voxel classification is performed with linear and quadratic discriminant classifiers with the use of MRI intensities, Gaussian smoothed intensities, gradient magnitude, Laplacian and distance features. Classifier performance is evaluated by direct voxel-wise comparison between MRI and histology for different combinations of features and classifiers.

\section{METHODS}

\subsection{Data}

Fifteen patients scheduled for carotid endarterectomy (CEA) were included in the study (5 TIA, 9 ischemic stroke, 1 asymptomatic). During CEA procedure the plaque was removed intact to keep its 3D shape as much as possible. The ex vivo specimens were scanned in a 3T clinical MRI scanner (Signa Excite, GE Healthcare, Milwaukee, USA) with high resolution. Four MRI contrast weightings were obtained: 3D-T1w gradient echo (GRE) (resolution 0.1x0.1 $1 \times 0.1 \mathrm{~mm}$ ), 2D-T1w spin echo (SE), 2D-T2w SE and 2D-PDw SE (all with a resolution of $0.1 \times 0.1 \times 0.6 \mathrm{~mm}$ ). To allow accurate quantification of calcification, the specimens were scanned with a $\mu \mathrm{CT}$ scanner (Skyscan 1072, Skyscan, Belgium, resolution $18 \times 18 \times 18 \mu \mathrm{m})$.

After imaging the specimens were decalcified and embedded in paraffin for histology processing. Every 1mm-interval axial slices of $5 \mu \mathrm{m}$ thickness were obtained. Staining was done with Elastica von Gieson (EvG, Merck, Germany), which allows visualization of cell nuclei, elastin and collagen fibers. On the original digitized histological slices, contours were manually drawn to segment the vessel wall into different regions. Contours for the lumen and outer vessel wall and for regions of necrotic tissue were drawn. These necrotic regions often contained lipid tissue, which makes these regions lipid-rich necrotic cores. Calcified areas were obtained from the $\mu \mathrm{CT}$. After standardization of the $\mu \mathrm{CT}$ images, calcifications were obtained by intensity thresholding at a value similar for all images. The remaining areas were considered to be fibrous tissue, resulting in three components (calcification, fibrous tissue and necrotic core) to be used for classification. 


\subsection{Registration}

The 3D registration procedure followed the pipeline developed in our group, to match in vivo CTA with histology via $\mu \mathrm{CT}$ and ex vivo $\mathrm{MRI}^{18}$. It includes multiple steps using both rigid and deformable registration and involves several manual annotations of anatomical landmarks, lumen and outer vessel wall contours. The schematic below illustrates the different stages in registration. The arrows point from the fixed image toward the moving image. Following it, a more detailed description of each transformation is given.

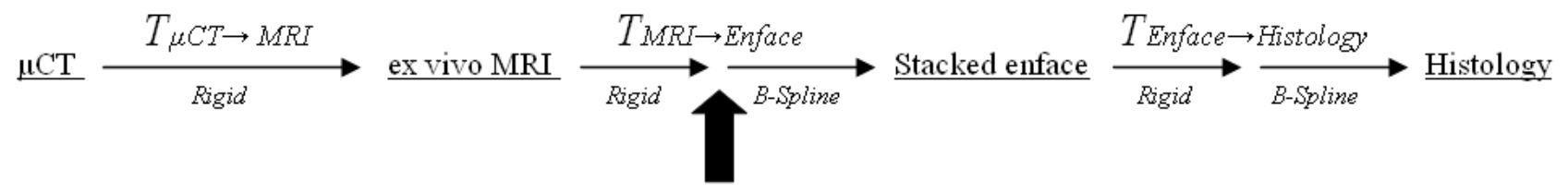

- Enface stacking: Digital photographs of the cutting plane during histology slicing were taken at every $1 \mathrm{~mm}$ interval. These 'enface' images were stacked by 2D in-plane rigid registrations to form a 3D volume. This was performed by point-based registration of manually annotated landmarks.

- $T_{\text {Enface } \rightarrow \text { Histology: }}$ The 2D histology slices were registered to the enface stack to obtain a 3D histology. This step allowed for non-rigid in-slice deformations by using a B-spline deformation model ${ }^{19}$. Manual annotations contained several landmarks and segmentations of the lumen and outer vessel wall. The landmarks were used for rigid initialization, followed by maximizing the mutual information ${ }^{20,21}$ (MI) between the original images, lumen segmentations and outer wall segmentations with the B-spline model. The resulting transformation was applied to the histology images, resulting in a '3D histology stack'.

- $T_{M R I \rightarrow E n f a c e:}$ This registration was initialized with a rigid registration based on annotated landmarks in the 'enface' and 3D-T1w MRI, followed by an automated rigid step using the 3D histology stack. This step made use of the same MI maximization as before, for which manual lumen and outer vessel wall segmentations were made on the MRI. The registration was refined by 3D B-spline deformable registration with the same similarity measure as used when optimizing $T_{\text {Enface } \rightarrow H i s t o l o g y}$. In this last stage, only in-plane deformations were allowed. The various MRI images were assumed to be aligned to each other, since the specimen did not move between acquisitions.

- $T_{\mu C T \rightarrow M R I}$ : For registration of the ex vivo MRI to the $\mu \mathrm{CT}$ a rigid registration without scaling was assumed, which was performed point-based using annotated landmarks in the $\mu \mathrm{CT}$ and 3D-T1w image.

All registration steps were performed using the registration toolbox elastix ${ }^{22}$. Classification was performed in the space indicated by the block arrow in the above given schematic. All images were transformed to this domain. This means that only the B-spline transformation part of $T_{M R I \rightarrow E n f a c e}$ was applied to the 3D histology stack, and that the (inverse) rigid transformation part was applied to the ex vivo MRI. The resolution in this domain was set to $0.017 \times 0.017 \times 1 \mathrm{~mm}$, which equals the resolution of the enface images. Examples of registered images for one specimen are shown in figure 1. 

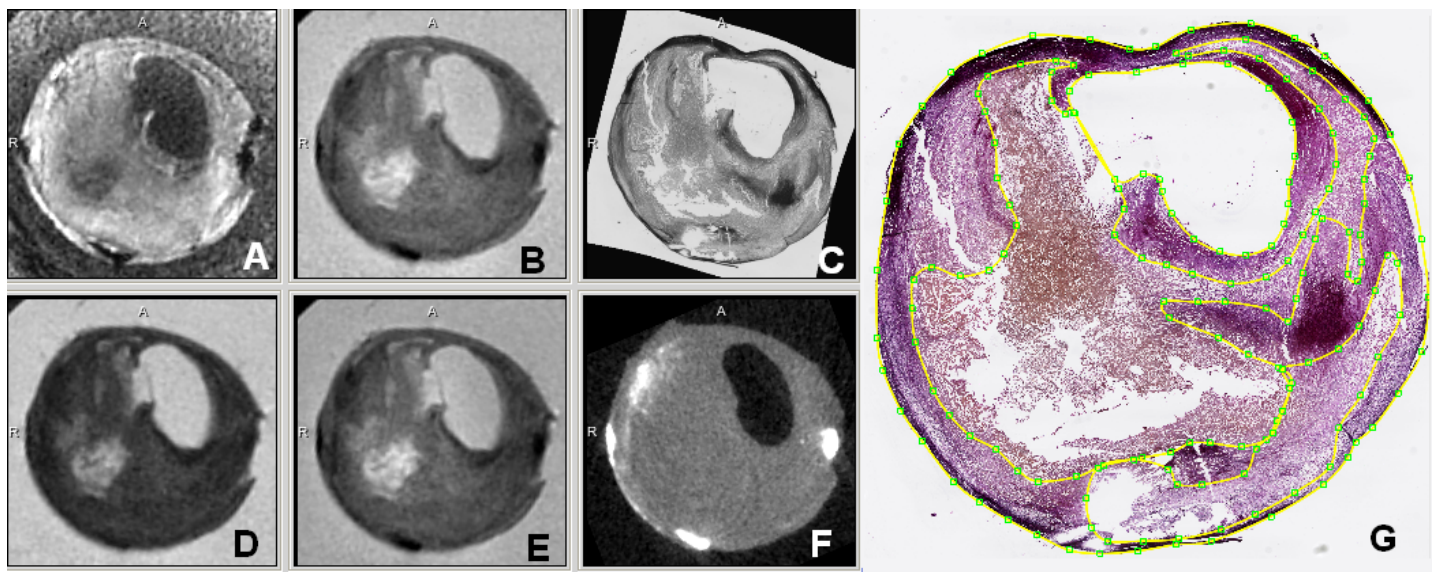

Figure 1: Example of a set of registered imaging data: (A) 3D-T1w, (B) T1w, (C) deformed histology, (D) T2w, (E) PDw and (F) $\mu \mathrm{CT}$ images at one location for one specimen. Also, the original EvG histology slice with manual annotations is shown $(\mathrm{G})$. Contours for the lumen and outer vessel wall, and necrotic regions are present.

\subsection{Classification}

To remove intensity gradients in the original MR images, nonparametric nonuniform intensity normalization ${ }^{23}$ (N3) was applied. For intensity normalization between patients, the images were scaled with respect to the average intensity of the fluid in the tube surrounding the plaque, which was set to 1000. To account for slight misregistrations, both in classifier training and testing, a morphological erosion with a disk structuring element was applied to the segmentations. For calcifications the diameter of the structuring element was set to 3 voxels, for fibrous and necrotic to 5 voxels, as the registration of $\mu \mathrm{CT}$ and MRI was assumed to be more accurate than that of histology and MRI. This erosion deleted $21 \%$ of the voxels in the original ground truth segmentations.

For every voxel the following features were obtained:

- Intensities in all four normalized MR images and after convolution with a Gaussian with $\sigma=1, \sigma=2$ and $\sigma=3$ times the voxelsize in the $3 \mathrm{DT} 1 \mathrm{~W}$ scan to obtain smoothed images

$$
G(x, y, z)=\frac{1}{\sqrt{2 \pi \sigma^{2}}{ }^{3}} e^{-\frac{x^{2}+y^{2}+z^{2}}{2 \sigma^{2}}}
$$

- The gradient magnitude of all smoothed images

$$
\text { GradientMagnitude }=\sqrt{\left(\frac{\partial f(x, y, z)}{\partial x}\right)^{2}+\left(\frac{\partial f(x, y, z)}{\partial y}\right)^{2}+\left(\frac{\partial f(x, y, z)}{\partial z}\right)^{2}}
$$

- The Laplacian of all smoothed images

$$
\text { Laplacian }=\frac{\partial^{2} f(x, y, z)}{\partial x^{2}}+\frac{\partial^{2} f(x, y, z)}{\partial y^{2}}+\frac{\partial^{2} f(x, y, z)}{\partial z^{2}}
$$

- The Euclidean distance to the lumen and to the outer vessel wall

This resulted in a total of 42 features. Calculation of the smoothed, gradient magnitude and Laplacian features was done before registration with the histology stack. The distances were calculated in the 2D slices after registration. The applied classifiers were linear (LDC) and quadratic (QDC) discriminant functions. Both assume the data is normally distributed, but LDC assumes equal covariance matrices for the three classes, while this is not assumed for QDC. The discriminant functions are as follows ${ }^{24}$ : 
LDC:

$\delta_{k}(x)=x^{T} \Sigma^{-1} \mu_{k}-\frac{1}{2} \mu_{k}^{T} \Sigma^{-1} \mu_{k}+\log \pi_{k}$

QDC:

$\delta_{k}(x)=-\frac{1}{2} \log \left|\Sigma_{k}\right|-\frac{1}{2}\left(x-\mu_{k}\right)^{T} \Sigma_{k}^{-1}\left(x-\mu_{k}\right)+\log \pi_{k}$

Here $k$ are the classes, $\mu_{k}$ the mean, $\Sigma_{k}$ the covariance matrix and $\pi_{k}$ the prior probability of class $k$ and $x$ the feature vector to classify.

The classifiers were evaluated in a leave-one-out manner in which one specimen was used for testing and the rest formed the training set. For all images in each training set, $1 \%$ of the voxels were randomly selected to train the classifiers. Both classifier development and evaluation was performed using the Matlab toolbox 'prtools' ${ }^{25}$, version 4.1.9

\section{RESULTS}

Only 12 of the 15 specimens were included in the study, because of low quality of histology ( 2 cases) or low MRI image quality ( 1 case). The number of included histology slices with corresponding MRI was $10.7 \pm 3.9$ per patient. The complete set of slices contained 5\% calcification, $63 \%$ fibrous tissue and $32 \%$ necrotic tissue.

The average leave-one-out accuracies for LDC and QDC with different combinations of features are shown in Table 1. To compare, random class assignment according to the class priors would result in an accuracy of $51 \%$. As can be seen, for the individual original MR images QDC gives a slightly higher accuracy than LDC, which is only significant for the PDw images (paired t-test, $\mathrm{p}<0.05$ ). For the other feature sets LDC performs better, which is significant for the Laplacian features and the total feature set (paired t-test, $\mathrm{p}<0.05$ ). A combination of the four original image intensities yields an accuracy of $62.8 \pm 13.3 \%$ when using LDC, which increases by adding subsequently smoothed images $(67.3 \pm 10.6 \%)$, distance features $(71.6 \pm 7.9 \%)$ and finally the gradient magnitude and Laplacian images $(72.5 \pm 7.7 \%)$. This last classifier performs significantly better than all other linear classifiers (paired t-test, $\mathrm{p}<0.05$ ).

Table 1: Accuracies for linear (LDC) and quadratic (QDC) classification for different combinations of features. The value between parentheses indicates the total number of features included in the feature set. * indicates a significant difference between LDC and QDC and $\uparrow$ indicates a significant difference with the classifier including all 42 features (paired t-test, $\mathrm{p}<0.05$ ), for either LDC or QDC.

\begin{tabular}{ccc}
\hline Included features & LDC & QDC \\
\hline 3D-T1w GRE (1) & $60.3 \pm 17.0 \% \dagger$ & $60.5 \pm 16.9 \%$ \\
T1w-SE (1) & $60.4 \pm 16.8 \% \dagger$ & $61.6 \pm 14.2 \%$ \\
T2w-SE (1) & $58.8 \pm 16.9 \% \dagger$ & $59.2 \pm 17.1 \%$ \\
PDw-SE (1) & $60.9 \pm 16.9 \% \dagger$ & $61.1 \pm 16.8 \% *$ \\
Gradient magnitude (12) & $57.1 \pm 14.7 \% \dagger$ & $54.0 \pm 7.3 \% \dagger$ \\
Laplacian (12) & $60.5 \pm 16.3 \% \dagger$ & $57.5 \pm 13.6 \% * \dagger$ \\
Distances (2) & $67.5 \pm 7.2 \% \dagger$ & $67.3 \pm 7.7 \%$ \\
4 original images (4) & $62.8 \pm 13.3 \% \dagger$ & $61.8 \pm 13.0 \% \dagger$ \\
4 original + smoothed images (16) & $67.3 \pm 10.6 \% \dagger$ & $64.1 \pm 12.3 \%$ \\
Original + smoothed images + distances (18) & $71.6 \pm 7.9 \% \dagger$ & $67.6 \pm 12.1 \%$ \\
Original + smoothed images + Gradient magnitude + Laplacian + distances (42) & $72.5 \pm 7.7 \%$ & $68.8 \pm 7.6 \% *$ \\
\hline
\end{tabular}

A more detailed overview of the performance of the best classifier (i.e. including all features and using LDC) on the different plaque types is shown below. Table 2 shows the confusion matrix of the different plaque components and Table 3 lists measures such as sensitivity, specificity, positive and negative predictive value, derived from Table 2 . The results for each tissue type in Table 3 are computed by considering the other two types together as one background class. For calcification a good sensitivity and specificity are obtained, while for fibrous tissue the specificity is lower and for necrotic tissue the sensitivity is lower. This indicates that the differentiation between fibrous and necrotic tissue is more difficult and, as seen in Table 2, often leads to the misclassification of necrotic tissue as fibrous tissue. Although 
calcification shows good sensitivity and specificity, the positive predictive value is only $52 \%$. The reason for this is that misclassification of small regions of fibrous or necrotic tissue as calcification is relatively large compared to the total volume of calcification present. Class accuracies are high for calcification and reasonable for fibrous and necrotic tissue. The total accuracy of $73.5 \%$ is larger than the $72.5 \%$ shown before, since in Table 3 all voxels are considered, while Table 1 lists the average classification accuracy per subject.

Table 2: Confusion matrix for linear classification based on all features. Values represent the percentage of the total amount of voxels, summed over all subjects.

\begin{tabular}{|c|c|c|c|c|c|}
\hline & $\begin{array}{l}\text { Classifier } \\
\text { Calcification }\end{array}$ & \multicolumn{2}{|c|}{ Classifier } & Total \\
\hline \multirow[t]{4}{*}{ Ground truth } & Calcification & $4.6 \%$ & $0.6 \%$ & $0.4 \%$ & $5.6 \%$ \\
\hline & Fibrous & $2.5 \%$ & $53.4 \%$ & $7.0 \%$ & $62.9 \%$ \\
\hline & Necrotic & $1.8 \%$ & $14.2 \%$ & $15.5 \%$ & $31.5 \%$ \\
\hline & Total & $8.9 \%$ & $68.2 \%$ & $22.9 \%$ & $100 \%$ \\
\hline
\end{tabular}

Table 3: Results for linear classification based on all features. For each component sensitivity, specificity, positive predictive value (PPV), negative predictive value (NPV), accuracy per class and the area under the ROC curve (AUC) are given. The last column gives the overall accuracy as a percentage of correctly classified voxels.

\begin{tabular}{cccccccc}
\hline & Sensitivity & Specificity & PPV & NPV & Class accuracy & AUC & Overall accuracy \\
\hline Calcification & $83 \%$ & $95 \%$ & $52 \%$ & $99 \%$ & $95 \%$ & 0.94 & $73.5 \%$ \\
Fibrous & $85 \%$ & $60 \%$ & $78 \%$ & $70 \%$ & $76 \%$ & 0.76 & \\
Necrotic & $49 \%$ & $89 \%$ & $68 \%$ & $79 \%$ & $77 \%$ & 0.72 & \\
\hline
\end{tabular}

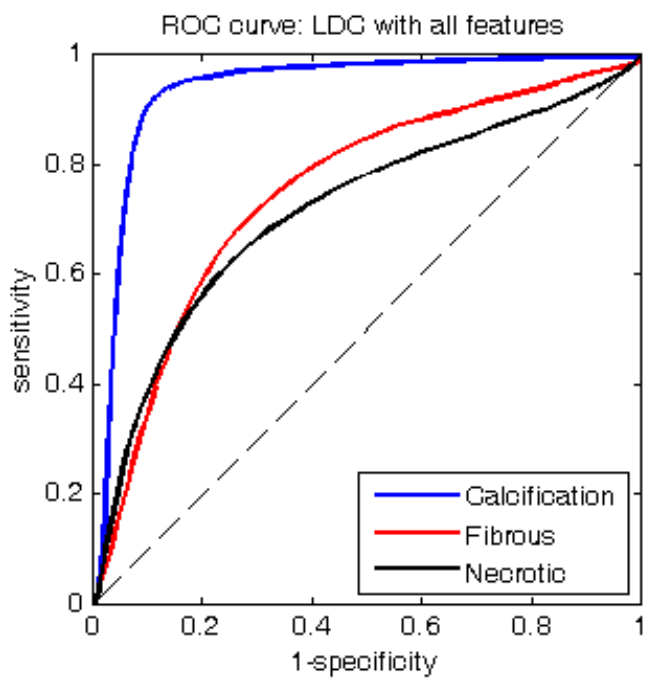

Figure 2: Receiver-Operating curves for linear classification with all features. Areas under the curve for calcification, fibrous and necrotic tissue are $0.94,0.76$ and 0.72 respectively. 

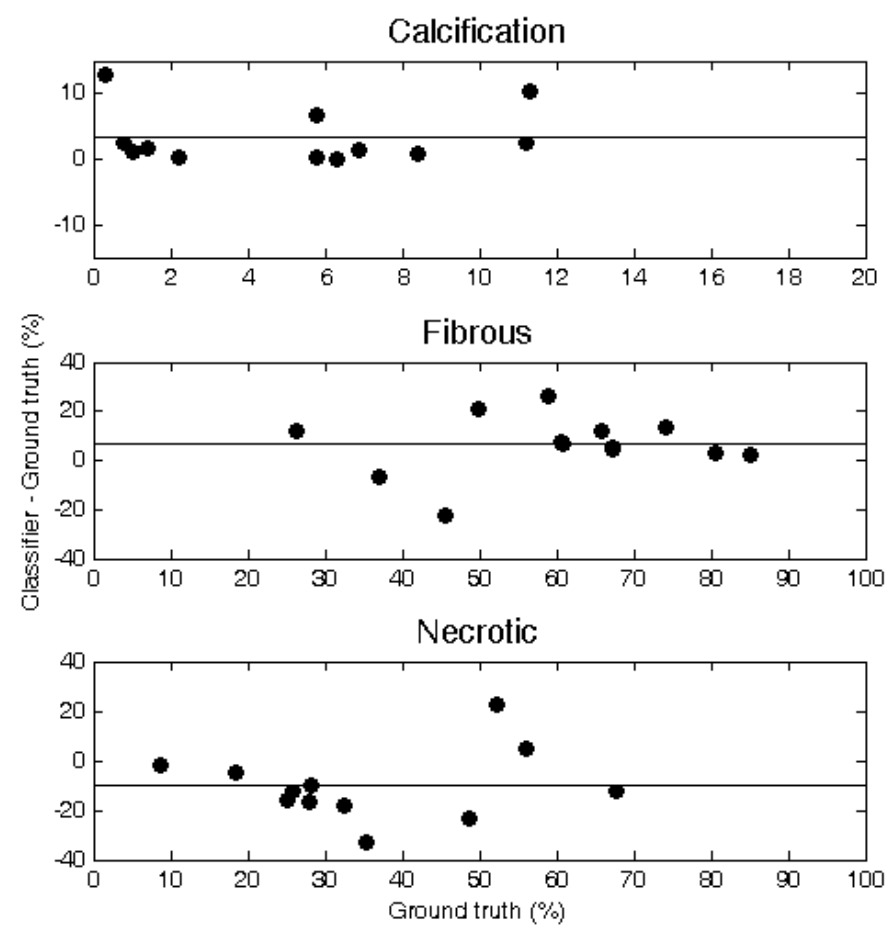

Figure 3: Three plots showing the difference in measured volume ( $\%$ of total plaque volume) per component between the classifier and the ground truth ( $\mu \mathrm{CT}$ and histology). The mean differences for calcification, fibrous and necrotic tissue are $3.4 \pm 4.3 \%, 6.6 \pm 12.7 \%$ and $-10.0 \pm 14.2 \%$ respectively, and indicated by the black line.

The Receiver-Operating curves (figure 2) also show that for calcification a good sensitivity and specificity can be reached, with lower areas under the curve for the other two components. The plots in figure 3 show the differences between the classifier and the ground truth, as a percentage of total plaque volume, for all three plaque components. It shows an average overclassification of calcification (3.4 $\pm 4.3 \%)$ and fibrous tissue $(6.6 \pm 12.7 \%)$ and underclassification of necrotic core $(-10.0 \pm 14.2 \%)$. For calcification the differences are around zero, with few exceptions that show larger overclassification due to imaging artifacts. For fibrous and necrotic tissue the differences may become relatively large compared to the ground truth value, due to the misclassification of large regions of necrotic core as fibrous tissue.

For illustration, figures 4 to 6 show examples of the classification results of three specific MRI slices. In figure 4 the classification is good, with an accuracy of $85 \%$. A small mistake is seen close to the lumen at the center, where a part of the fibrous cap is classified as necrotic. This is probably caused by a black image artifact in the lumen that can be seen in the MR images and is probably caused by the presence of an air bubble. In addition, a fibrous structure that separates two necrotic regions is not detected, as it is very small. Figure 5 shows one of the slices with large errors (accuracy $53 \%$ ). Three regions of necrotic tissue are completely missed and classified as fibrous tissue. One region shows disruption in the MRI, but for the other two the intensity contrast seems low. When segmenting the histology, the observer expected the disrupted area to be disrupted due to histology slicing, so after MRI scanning. It happens more often that necrotic regions are completely missed by the automatic classification, but this usually concerns small structures. In larger necrotic regions, as in this example, in most cases a part of it is correctly detected as necrotic. Figure 6 shows an example with an accuracy of $60 \%$, in which calcification is overclassified due to an imaging artifact. This artifact is probably caused by the presence of air in the specimen. The real calcification is, however, accurately found. 


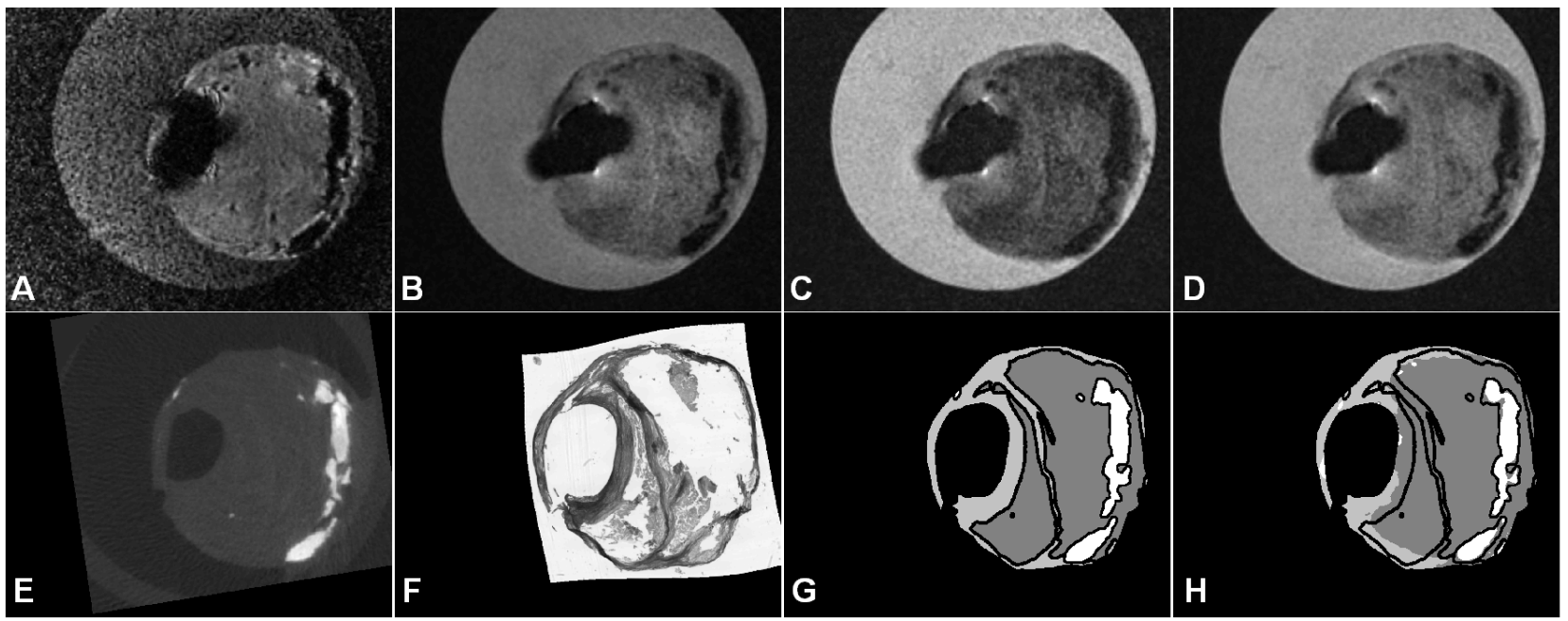

Figure 4: An example of a well-classified slice (85\% accuracy), showing 3DT1W (A), T1W (B), T2W (C) and PDW (D) images, $\mu \mathrm{CT}(\mathrm{E})$, histology $(\mathrm{F})$, the ground truth segmentation $(\mathrm{G})$ and the classifier result $(\mathrm{H})$. In $\mathrm{G}$ and $\mathrm{H}$ calcification is white, fibrous tissue light gray and necrotic tissue dark gray. Calcification is accurately detected. A small part of the fibrous cap is classified as necrotic tissue, probably caused by the black artifact that is visible in A-D.

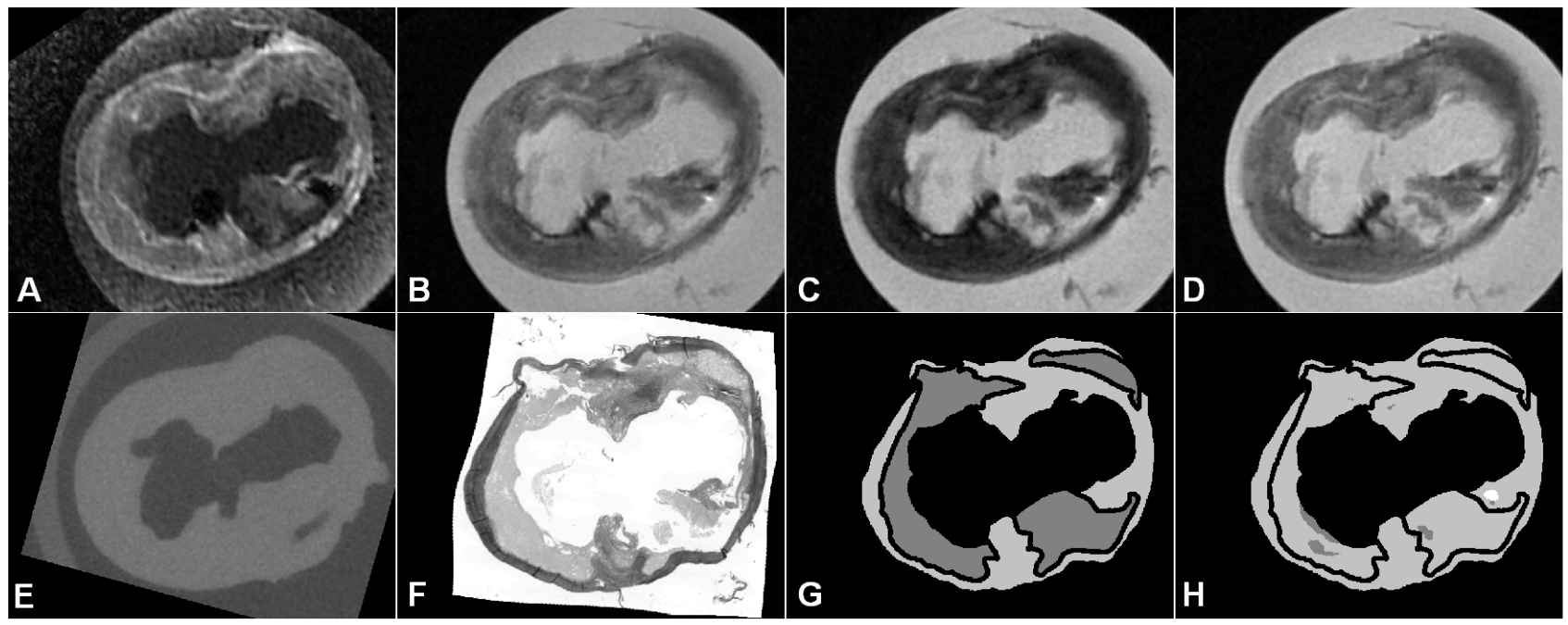

Figure 5: An example of a slice with large misclassifications (53\% accuracy), showing 3DT1W (A), T1W (B), T2W $(C)$ and PDW (D) images, $\mu \mathrm{CT}(\mathrm{E})$, histology $(\mathrm{F})$, the ground truth segmentation $(\mathrm{G})$ and the classifier result $(\mathrm{H})$. In $\mathrm{G}$ and $\mathrm{H}$ calcification is white, fibrous tissue light gray and necrotic tissue dark gray. All three necrotic regions are missed. One is disrupted in the histology, and the other two show low intensity contrast in the MRI. 

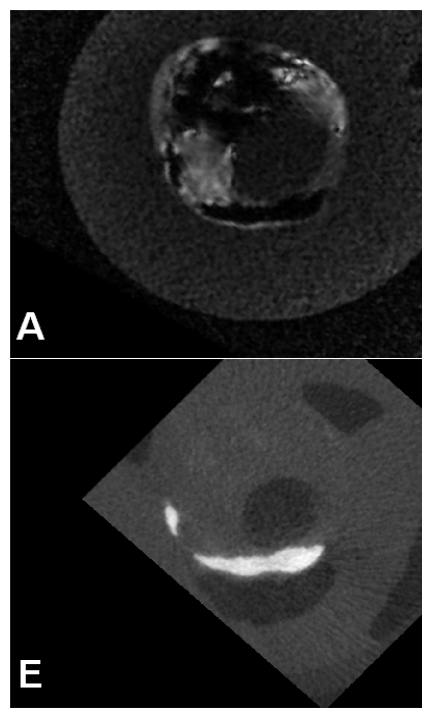
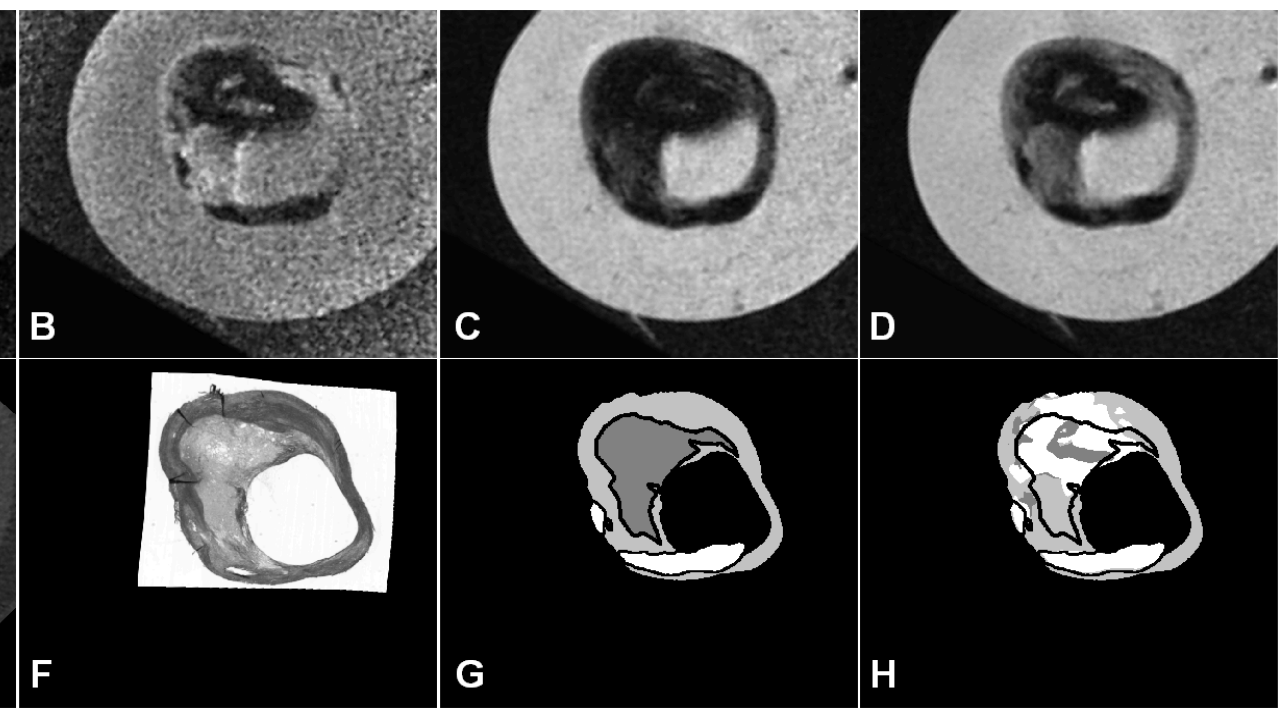

Figure 6: An example of a slice with overclassification of calcification (60\% accuracy), showing 3DT1W (A), T1W (B), T2W (C) and PDW (D) images, $\mu \mathrm{CT}(\mathrm{E})$, histology $(\mathrm{F})$, the ground truth segmentation $(\mathrm{G})$ and the classifier result $(\mathrm{H})$. In $\mathrm{G}$ and $\mathrm{H}$ calcification is white, fibrous tissue light gray and necrotic tissue dark gray. The region that shows up dark in the MRI and is not visible in the $\mu \mathrm{CT}$ is an artifact caused by the presence of water and is therefore by the classifier recognized as calcification, which also is dark on MRI.

\section{DISCUSSION}

Classification of carotid plaque components based on training and validation on MRI images registered to a 3D histology stack, is presented with reasonable accuracy. The evaluation of classifiers in which the original image intensities were combined with other features showed improved classification accuracy. Firstly, adding smoothed images had a positive effect, most likely due to the reduction of noise and inclusion of some information of the surrounding tissue. In addition, as in earlier publications, the distance to the lumen and outer vessel wall improved accuracy ${ }^{16}$. This can be explained by the fact that lipid or necrotic tissue usually occurs in the center of plaques and fibrous tissue near the boundaries. Gradient magnitude and Laplacian features were expected to be especially useful in detecting small structures as calcifications, and led to a small, but statistically significant improvement. In our results their inclusion indeed led to a better accuracy for calcification, but since this component was already accurately classified without these features and was the smallest component in the dataset, the effect was small. The results for single MRI contrast weightings, gradient magnitude and Laplacian images, and the two distances were shown for more insight in the behavior of these separate features.

For the best classifier (LDC with all features), accuracy was good for calcification (95\%), however, only reasonable for necrotic (77\%) and fibrous tissue (76\%). A reason might be that these components have a less clearly defined intensity profile in the used MR images. Histograms show that there is a large overlap in intensity between fibrous and necrotic tissue in all four MRI contrasts weightings. In addition, registration of ex vivo MRI to $\mu \mathrm{CT}$, which yielded the reference for calcification is usually more accurate than that of ex vivo MRI to histology due to deformations that occur during slicing. However, visual inspection does not suggest large misregistrations for histology. The largest mistakes that are currently made in classification of calcification are due to the occurrence of black artifacts that are classified as calcification. In future classifiers attempts may be made to differentiate these artifacts from real calcifications.

The presented results are comparable to previous studies that performed ex vivo plaque classification with voxelwise analysis $^{10,12,17}$. These studies used only the original intensity MR images and had resolutions slightly lower than in the present study. Clarke et al. yielded an overall accuracy of $73.5 \%{ }^{10}$ when 8 contrast weightings were used, or $78 \%{ }^{12}$ when either these 8 scans or a combination of $\mathrm{T} 1 \mathrm{~W}, \mathrm{~T} 2 \mathrm{~W}$ and diffusion weighting (DW) was used. A combination of $\mathrm{T} 1 \mathrm{~W}$, T2W and PDW as in the present study had in that study an accuracy of $67 \%{ }^{12}$, which is better than the $63 \%$ for our classifier that used only the original images. Another study that obtained errors of $>40 \%$ when five components were distinguished and $20-30 \%$ for three components, also yielded slightly better results when PDW images were replaced by DW images ${ }^{17}$. In terms of volumetric analysis, Karmonik et al. looked at the most abundant plaque component per slice 
and obtained smaller absolute differences than we (calcifications: $1.5 \pm 1.4 \%$, fibrous $4.0 \pm 2.8 \%$ and lipid: $2.4 \pm$ $2.2 \%)^{15}$. However, these studies all used manual selection of individual corresponding MRI and histology slices and included fewer slices per subject. This may have led to an overestimation of the accuracy, as such selected histology and MRI slices may look more similar than after objective 3D registration and only the few slices with best histology and MRI quality were included. The degree of bias that is introduced by manually selecting and matching histology to MRI has still to be evaluated, but might be an explanation for the differences. However, it remains difficult to compare results, as they are obtained on different datasets with different MRI protocols, image quality and possibly other factors.

\section{CONCLUSION}

We presented a new system for the classification of calcification, fibrous and necrotic tissue in ex vivo carotid specimens. Our approach uses 3D registration of MRI with a histology stack to train and evaluate classifiers. In addition, next to original MRI intensities also smoothed intensities, distances to the lumen and outer vessel wall, the gradient magnitude and Laplacian were included as features. This method yields reasonable results. Although the differentiation between fibrous and necrotic tissue lacks sensitivity and specificity, calcification can be segmented with high accuracy.

\section{ACKNOWLEDGEMENTS}

This research was performed within the framework of CTMM, the Center for Translational Molecular Medicine (www.ctmm.nl), project PARISk (grant 01C-202), and supported by the Netherlands Heart Foundation. Marleen de Bruijne, Stefan Klein and Wiro Niessen were financially supported by the Netherlands Organisation for Scientific Research (NWO). Harald Groen was financially supported by the Interuniversity Cardiology Institute of the Netherlands.

\section{REFERENCES}

[1] Petty, G. W., Brown, R. D., Whisnant, J. P., Sicks., J. D., O'Fallon, W. M. and Wiebers, D. O. , “Ischemic stroke subtypes - A population-based study of incidence and risk factors," Stroke 30, 2513-2516 (1999).

[2] Seeger, J., M., Barratt, E., Lawson, G., A. and Klingman, N., "The relationship between carotid plaque composition, plaque morphology and neurologic symptoms", Journal of surgical research 58(3), 330-336 (1995).

[3] Shalaan, W. E., Cheng, H., Gewertz, B., McKinsey, J. F., Schwartz, L. B., Katz, D., Cao, D., Desai, T., Glagov S. and Bassiouny, H. S., "Degree of carotid plaque calcification in relation to symptomatic outcome and plaque inflammation," Journal of Vascular Surgery 40(2), 262-269 (2004).

[4] Yuan, C., Zhang, S., Polisser, N. L., Echelard, D., Ortiz, G., Davis, J. W., Ellington, E., Ferguson, M. S. and Hatsukami, T. S., "Identification of fibrous cap rupture with magnetic resonance imaging is highly associated with recent transient ischemic attack or stroke," Circulation 105, 181-185 (2002).

[5] Saam, T., Ferguson, M., S., Yarnykh, V. L., Takaya, N., Xu, D., Polissar, N. L., Hatsukami, T. S. and Yuan, C., "Quantitative evaluation of carotid plaque composition by in vivo MRI," Arterioscler Thromb Vasc Biol 25, 234-239 (2005).

[6] Toussaint, J. F., LaMuraglia, G. M., Southern, J. F., Fuster, V. and Kantor, H. L., "Magnetic resonance images lipid, fibrous, calcified, hemorrhagic, and thrombotic components of human atherosclerosis in vivo," Circulation 94(5), 932-938 (1996).

[7] Yuan, C., Mitsumori, L. M., Ferguson, M. S., Polissar, N. L., Echelard, D., Ortiz, G., Small, R., Davies, J. W., Kerwin, W. S. and Hatsukami, T. S., "In vivo accuracy of multispectral magnetic resonance imaging for identifying lipid-rich necrotic cores and intraplaque hemorrhage in advanced human carotid plaques," Circulation 104, 2051-2056 (2001).

[8] Morrisett, J., Vick, W., Sharma, R., Lawrie, G., Reardon, M., Ezell, E., Schwartz, J., Hunter, G. and Gorenstein, D., "Discrimination of components in atherosclerotic plaques from human carotid endarterectomy specimens by magnetic resonance imaging ex vivo," Magnetic Resonance Imaging 21, 465-474 (2003).

[9] Shinnar, M., Fallon, J. T., Wehrli, S., Levin, M., Dalmacy, D., Fayad, Z. A., Badimon, J. J., Harrington, M., Harrington, E. and Fuster, V., "The diagnostic accuracy of ex vivo MRI for human atherosclerotic plaque characterization," Arterioscler Thromb Vasc Biol 19, 2756-2761 (1999). 
[10] Clarke, S. E., Hammond, R. R., Mitchell, J. R. and Rutt, B. K., "Quantitative assessment of carotid plaque composition using multicontrast MRI and registered histology", Magnetic Resonance in Medicine 50, 11991208 (2003).

[11] Adame, I. M., van der Geest, R. J., Wasserman, B. A., Mohamed, M. A., Reiber, J. H. C. and Lelieveldt, B. P. F., "Automatic segmentation and plaque characterization in atherosclerotic carotid artery MR images," MAGMA 16, 227-234 (2004).

[12] Clarke, S. E., Beletsky, V., Hammon, R. R., Hegele, R. A. and Rutt, B. K., "Validation of automatically classified magnetic resonance images for carotid plaque compositional analysis," Stroke 37, 93-97 (2006).

[13] Hofman, J. M. A., Branderhorst W. J., ten Eikelder, H. M. M., Cappendijk, V. C., Heeneman, S., Kooi, M. E., Hilbers, P. A. J. and ter Haar Romeny, B. M., "Quantification of atherosclerotic plaque components using in vivo MRI and supervised classifiers," Magnetic Resonance in Medicine 55, 790-799 (2006).

[14] Itksovitch, V. V., Samber, D. D., Mani, V., Aguinaldo, J. G. S., Fallon, J. T., Tang, C. Y., Fuster, V. and Fayad, Z. A., "Quantification of human atherosclerotic plaques using spatially enhanced cluster analysis of multicontrast-weighted magnetic resonance images," Magnetic Resonance in Medicine 52, 515-523 (2004).

[15] Karmonik, C., Basto, P., Vickers, K., Martin, K., Reardon, M. J., Lawrie, G. M. and Morrisett, J. D., "Quantitative segmentation of principal carotid atherosclerotic lesion components by feature space analysis bassed on multicontrast MRI at 1.5 T," IEEE Transactions on Biomedical Engineering 56(2), 352-360 (2009).

[16] Liu, F., Xu, D., Ferguson, M. S., Chu, B., Saam, T., Takaya, N., Hatsukami, T. S., Yuan, C. and Kerwin, W. S., "Automated in vivo segmentation of carotid plaque MRI with morphology-enhanced probability maps," Magnetic Resonance in Medicine 55, 659-668 (2006).

[17] Ronen, R. R., Clarke, S. E., Hammond, R. R., and Rutt, B. K., "Carotid plaque classification: Defining the certainty with which plaque components can be differentiated," Magnetic Resonance in Medicine 57, 874-880 (2007).

[18] Groen, H. C., van Walsum, T., Rozie, S., Klein, S., van Gaalen, K., Gijsen, F. J., Wielopolski, P. A., van Beusekom, H. M., de Crom, R., Verhagen, H. J., van der Steen, A. F., van de Lugt, A., Wentzel, J. J., and Niessen, W. J., "Three-dimensional registration of histology of human atherosclerotic carotid plaques to in vivo imaging", Journal of Biomechanics 43, 2087-2092 (2010).

[19] Rueckert, D., Sonoda, L. I., Hayes, C., Hill, D. L., Leach, M. O., Hawkes, D. J., "nonrigid registration using free-form deformations: application to breast MR images," IEEE Transactions on Medical Imaging 18(8), 712721 (1999).

[20] Viola, A. and Wells, W. M., "Alignment by maximization of mutual information," International Journal of Computer Vision 24(2), 137-154 (1997).

[21] Thevenaz, P. and Unser, M., "Optimization of mutual information for multiresolution image registration," IEEE Transactions on Image Processing 9(12), 2083-2099 (2000).

[22] Klein, S., Staring, M., Murphy, K., Viergever, M. A. and Pluim, J. P. W. "elastix: a toolbox for intensity based medical image registration," IEEE Transactions on Medical Imaging 29(1), 196 - 205 (2010).

[23] Sled, J. G., Zijdenbosch, A. P. and Evans, A. C., "A nonparametric method for automatic correction of intensity nonuniformity in MRI data," IEEE Transactions on Medical Imaging 17(1), 87-97 (1998).

[24] Hastie, T., Tibshirani, R. and Friedman, J., [The elements of statistical learning: Data mining, inference, and prediction, second edition], Springer, New York (2009).

[25] Duin, R. P. W., Juszczak, P., Paclik, P., Pekalska, E., de Ridder, D., Tax, D. M. J. and Verzakov, S., "PRTools4.1, A Matlab toolbox for pattern recognition," Delft University of Technology, 2007. 